\title{
ANÁLISE DOS CUSTOS DIRETOS E INDIRETOS DO CONSULTÓRIO ODONTOLÓGICO NA UNIDADE DE SAÚDE DA FAMÍLIA NOBRE, PAULISTA-PE
}

\author{
E. S. Q. ESTIMA ${ }^{1}$, A. H. B. ARAÚJO ${ }^{1}$, A.V. A. SANTOS $^{1}$, M. M.M. de CARVALHO ${ }^{1}$, R. \\ S. C. AGUIAR ${ }^{1}$, K. F. MARTINS ${ }^{1}$
}

${ }^{1}$ Universidade Federal de Pernambuco, Centro de Ciências Sociais Aplicadas, Departamento
de Economia, Programa de Pós Graduação em gestão e Economia da Saúde

E-mail para contato: manuqueirozz@hotmail.com

RESUMO- A Estratégia Saúde da Família (ESF) é o objeto principal da Atenção Primária de Saúde. É composta por equipe multiprofissional que foi acrescida desde janeiro de 2004 dos profissionais de saúde bucal, norteada por diretriz elaborada pelo Ministério da Saúde visando uma reorganização da atenção em saúde bucal e a promoção da qualidade de vida. Os custos no setor público são financiados através do orçamento público da União, Estados, Municípios e Distrito Federal, já as despesas são realizadas através de transferências aos prestadores de serviços aos estados e municípios. A atenção básica por sua vez, é financiada pelo Piso da Atenção Básica fixo e variável. O trabalho tem por objetivo analisar os custos diretos e indiretos do Consultório Odontológico da Unidade de Saúde da Família Nobre do Município de Paulista-PE através de um estudo retrospectivo, descritivo com análise de dados de base documental e com abordagem quantitativa e o método de custeio utilizado foi o de absorção. Na análise de custeio por absorção, foram vistos os principais procedimentos odontológicos como: extração, restauração e profilaxia. Observou-se então que dentre os custos diretos, o maior encontrado foi relacionado com o procedimento de restauração e o menor de profilaxia. Logo, percebe-se que a alocação de recursos e custos para procedimentos curativos são maiores e que o repasse Fundo a Fundo é insuficiente para atender às necessidades do consultório odontológico.

Palavras-chave: Atenção básica, custeio por absorção, gestão.

ABSTRACT The Family Health Strategy (ESF) is the main object of Primary Health Care. It is composed of a multiprofessional team that has been added since January 2004 of oral health professionals, guided by a guideline elaborated by the Ministry of Health aiming at a reorganization of care in oral health and the promotion of quality of life. The costs in the public sector are financed through the public budget of the Union, States, Municipalities and Federal District, and expenses are made through transfers to service providers to the states and municipalities. The basic attention, in turn, is financed by the Fixed and Variable Basic Attention Floor. The objective of this study was to analyze the direct and indirect costs of the Dentistry Office of the Family Health Unit of the Municipality of Paulista-PE through a retrospective, descriptive study with documentary data analysis and with a quantitative approach and the costing method used was that of absorption. In the absorption cost analysis, the main dental procedures were seen: 
extraction, restoration and prophylaxis. It was observed that among the direct costs, the largest found was related to the restoration procedure and the lesser prophylaxis. Therefore, it is noticed that the allocation of resources and costs for curative procedures are greater and that the Fund to Fund transfer is insufficient to meet the needs of the dental office.

Keywords: Basic care, absorption costing, management.

\section{INTRODUÇÃO}

A Estratégia Saúde da Família (ESF) é o objeto principal da Atenção Primária de Saúde, e atende como primeiro nível do sistema local de saúde, é composta por equipe multiprofissional que possui, no mínimo, médico generalista ou especialista em saúde da família ou médico de família e comunidade, enfermeiro generalista ou especialista em saúde da família, auxiliar ou técnico de enfermagem e Agentes Comunitários de Saúde (ACS). Pôde-se acrescentar a esta composição a partir de janeiro de 2004, como parte da equipe multiprofissional, os profissionais de saúde bucal (ou Equipe de Saúde BucalESB): cirurgião-dentista generalista ou especialista em saúde da família, auxiliar e/ou técnico em Saúde Bucal, norteada por diretriz elaborada pelo Ministério da Saúde visando uma reorganização da atenção em saúde bucal e a promoção da boa qualidade de vida. (DAB, 2016).

Trata-se, portanto, de uma estratégia que envolve a comunidade, e coloca as equipes multiprofissionais mais perto dos domicílios, das famílias e das comunidades. Esta proposta luta para substituir a forma de pensar e praticar saúde, transformando o tradicional modelo sanitário brasileiro, médico, medicamentoso, curativo e individual que tem no hospital o lócus de solução de todo e qualquer problema de saúde, em modelo coletivo, multi e interprofissional.

Os custos no setor público são financiados através do orçamento público da União, Estados, Municípios e Distrito Federal. Já as despesas do MS são realizadas através de transferências aos prestadores de serviços e aos estados e municípios. A atenção básica por sua vez, é financiada pelo Piso da Atenção Básica fixo e variável (MENDES, 2005).

Sendo assim, este trabalho tem como objetivo principal analisar os custos do Consultório Odontológico em uma Unidade de saúde da Família localizado no Município de Paulista-PE, à luz da literatura da Contabilidade de Custos nos Serviços de Saúde, a fim de identificar os gastos, os custos fixos e variáveis e as despesas deste consultório para o SUS diante dos procedimentos de profilaxia, restauração e extração.

Logo, percebe-se que o custo da atenção à saúde se torna cada vez mais elevado, mesmo nos países mais ricos e, ainda assim, os recursos continuam sempre escassos frente ao crescente volume das necessidades em saúde, o que torna cada vez mais necessário o conhecimento sobre o gerenciamento dos custos, para que as produções dos serviços sejam mais eficazes, e que sejam elaboradas alternativas a fim de otimizar o desempenho das equipes, possibilitando melhores condições de trabalho para os profissionais e os gestores, e consequentemente levar melhores ações de saúde para a comunidade.

\section{MATERIAIS E MÉTODOS}

Estudo retrospectivo, descritivo de base documental com abordagem quantitativa, realizado no serviço de odontologia da Unidade de Saúde da Família Nobre localizado no município do Paulista - PE. Segundo dados do IBGE, o município possui 43 USF`s, dessas, 24 possuem Equipe de Saúde Bucal cadastradas. O serviço é responsável por realizar ações de promoção e prevenção de saúde e procedimentos básicos ambulatoriais. 
Realiza-se em média 256 atendimentos ambulatoriais mensalmente. Foram analisados os procedimentos mais frequentes, sendo eles restauração em resina composta, extração e profilaxia, relacionados com as principais variáveis envolvidas, sendo elas materiais, salários, energia elétrica e depreciação. O método de custeio utilizado foi o de absorção, que para SOUSA (2015) representa o instrumento mais clássico de gestão de custos nas mais diversas instituições, inclusive nas hospitalares podendo se rduzir custor e melhorar a qualidade e agilidade no atendimento.

ANDRADE (2000), refere que esse critério é utilizado pela contabilidade financeira (de uso geral) e conceitua os itens de custo como diretos e indiretos.

Os custos indiretos são calculados através de bases de rateio como: gastos com energia elétrica, água, telefone, depreciação, impostos e outros que não podem ser claramente identificados e os diretos são aqueles diretamente aplicados e são alocados aos departamentos segundo sua utilização LAGIOIA (2016).

Esquema básico do custeio por absorção segundo LEONE (2001): $1^{\circ}$ passo: A separação entre custos e despesas; $2^{\circ}$ passo: Apropriação dos custos diretos; $3^{\circ}$ passo: Apropriação dos custos indiretos, através do critério de rateio.

Por esse processo, as despesas são reconhecidas no momento de sua incorrência (utilização), enquanto os custos estarão relacionados aos serviços e aos medicamentos estocados e consumidos.

Os dados foram coletados através de análise documental e do serviço e entrevistas, pois a Secretaria de Saúde não possuía um sistema de análise de custos. Foi utilizado o programa Microsoft Excel 2010 para consolidação dos dados. A análise das informações contidas nas planilhas deu-se através da interpretação dos valores obtidos.

\section{RESULTADOS E DISCUSSÃO}

O método de custeio por absorção conceitua os itens de custos como diretos e indiretos, e atribui a cada Unidade ou centros de custos, os custos aplicados diretamente, sendo rateados os indiretos.

$\mathrm{Na}$ análise dos custos diretos relacionados com os materiais, o procedimento de restauração teve um total de $\mathrm{R} \$ 957,34$; extração, $\mathrm{R} \$ 431,71$ e profilaxia, $\mathrm{R} \$ 25,23$; além disso, tiveram gastos materiais gerais (outros procedimentos e finalidades) no valor de $\mathrm{R} \$ 3.688,00$, totalizando $\mathrm{R} \$ 5.102,63$ de custos materiais como mostra na tabela 1 . Não foram encontrados custos indiretos.

Tabela 1. Análise de custeio por absorção do consultório odontológico na Unidade de Saúde da Família Nobre, Paulista - PE.

\begin{tabular}{|c|c|c|c|c|c|c|}
\hline & \multicolumn{3}{|c|}{ Custos Diretos } & \multirow[t]{2}{*}{ Outros } & \multirow{2}{*}{$\begin{array}{l}\text { Custos } \\
\text { Indiretos }\end{array}$} & \multirow[t]{2}{*}{ Total } \\
\hline & Restauração & Extração & Profilaxia & & & \\
\hline Materiais & 957,34 & 431,71 & 25,23 & $3.688,35$ & - & $5.102,63$ \\
\hline Salários & $2.000,00$ & 500,00 & 410,00 & & $2.339,89$ & $5.249,89$ \\
\hline $\begin{array}{l}\text { Energia } \\
\text { elétrica }\end{array}$ & 16,26 & 4,07 & 3,33 & & 17,00 & 40,66 \\
\hline Depreciação & - & - & - & & 264,65 & 264,65 \\
\hline Total & $2.973,61$ & 935,78 & 438,56 & $3.688,35$ & $2.621,53$ & $10.657,83$ \\
\hline
\end{tabular}

Quanto aos custos diretos relacionados aos salários, restauração teve um total de $\mathrm{R} \$ 2.000,00$; extração, $\mathrm{R} \$ 500,00$ e profilaxia, $\mathrm{R} \$ 410,00$. Os custos indiretos foram de 
$\mathrm{R} \$ 2.339,89$, totalizando $\mathrm{R} \$ 5.249,89$ de custos salariais como demonstra a tabela 2.

Tabela 2. Análise de custeio por absorção - Custos com Salários do consultório odontológico na Unidade de Saúde da Família Nobre, Paulista - PE

\begin{tabular}{|c|c|c|}
\hline \multicolumn{2}{|c|}{ Salários } \\
\hline Indiretos & & \\
\hline Serviços Gerais & 880 & 73,33 \\
\hline Recepcionista & 880 & 176,55 \\
\hline Salário dentista + auxiliar (outros) & & 2090,00 \\
\hline Diretos & & \\
\hline Restauração & $2.000,00$ & \\
\hline Extração & 500,00 & $2.910,00$ \\
\hline Profilaxia Total & 410,00 & $\mathbf{5 . 2 4 9 , 8 9}$ \\
\hline \multicolumn{2}{c}{ Tot } \\
\hline
\end{tabular}

Em relação a energia elétrica, restauração teve um custo total de $\mathrm{R} \$ 16,26$; extração, $R \$ 4,07$ e profilaxia, $R \$ 3,33$. Os custos indiretos foram de $R \$ 17,00$, totalizando $\mathrm{R} \$ 40,66$ de custos com energia elétrica como refere na Tabela 1.

Analisando a depreciação dos equipamentos utilizados nos procedimentos, não foram encontrados custos diretos, mas os indiretos tiveram um total de R $\$ 264,65$. Quando somados todos os custos por absorção, o total foi de $\mathrm{R} \$ 10.657,83$ exemplificado na tabela 3.

Tabela 3. Análise de custeio por absorção com alocação de custos proporcional aos custos diretos por procedimento do consultório odontológico na Unidade de Saúde da Família Nobre, Paulista - PE

\begin{tabular}{|l|l|l|l|l|l|l|}
\hline & \multicolumn{2}{l}{ Custos Diretos } & Outros & \multicolumn{2}{l|}{ Custos Indiretos } & Total \\
\hline & $\$$ & $\%$ & 3688,35 & $\$$ & $\%$ & $\mathbf{3 6 8 8 , 3 5}$ \\
\hline Restauração & $2.973,61$ & 68,39 & & $1.792,86$ & 68,39 & $4.766,47$ \\
\hline Extração & 935,78 & 21,52 & & 564,15 & 21,52 & $1.499,93$ \\
\hline Profilaxia & 438,56 & 10,09 & & 264,51 & 10,09 & 703,08 \\
\hline Total & $\mathbf{4 . 3 4 7 , 9 4}$ & $\mathbf{1 0 0}$ & $\mathbf{3 6 8 8 , 3 5}$ & $\mathbf{2 . 6 2 1 , 5 3}$ & $\mathbf{1 0 0}$ & $\mathbf{1 0 . 6 5 7 , 8 3}$ \\
\hline
\end{tabular}

Logo o procedimento de prevenção mostra então ter um custo menor para a unidade. De acordo com o Ministério da Saúde, a Estratégia Saúde da Família surgiu com o intuito de reorganizar a atenção básica no Brasil, ampliando a resolutividade na situação de saúde das pessoas e coletividade, levando a uma importante relação de custo-efetividade, diminuindo assim, complicações e demandas nas atenções secundária e terciária de saúde (BRASIL, 2008).

\section{CONCLUSÃO}

Conclui-se assim, que o procedimento de prevenção tem um custo menor do que os procedimentos curativos para a Atenção Básica. Porém, de acordo com a análise dos dados apresentados, percebe-se que a alocação de recursos e custos para procedimentos 
curativos, são maiores.

Diante disso vê-se a importância e a necessidade de os gestores do setor público expandirem o conhecimento na área de gestão de custos, pois além de ajuda-los nas tomadas de decisões, haverá uma melhor aplicabilidade dos recursos escassos e consequentemente alcançarão a excelência no atendimento.

\section{REFERÊNCIAS}

ANDRADE, M. Custos na atividade hospitalar. Temas contábeis em destaque. Conselho Regional do Estado de São Paulo; coordenação José Barbosa da Silva Júnior. São Paulo: Atlas, 2000 (coleção seminários CRC/SP/IBRACON

LEONE, G. S. G. Custos- Planejamento, Implantação e Controle.3 Edição, São Paulo: Atlas, 2000.

BRASIL, Ministério da Saúde. Portaria no 2488 de 21 de Outubro de 2011. Política Nacional de Atenção Básica, estabelecendo a revisão de diretrizes e normas para a organização da Atenção Básica, para a Estratégia Saúde da Família (ESF) e o Programa de Agentes Comunitários de Saúde (PACS). Disponível em: $<$ http://bvsms.saude.gov.br/bvs/saudelegis/gm/2011/prt2488_21 10 2011.html $>$ Acess o em: 21/10/2016.

LAGIOIA, U. I Curso De Especialização Em Saúde Para Profissionais Da Área Hospitalar DMS/UFPE: Gestão De Sistemas De Custos. Recife, 2016. (Apostila).

MATOS, A. J. Gestão de Custos Hospitalares: Técnicas, análise e tomada de decisão. São Paulo: Editora STS, 2002

MENDES, A; MARQUES, R. M. O financiamento da Atenção Básica e da Estratégia Saúde da Família no Sistema Único de Saúde. Saúde Debate, Rio de Janeiro, v. 38, n. 103, p. 900-916, OUT-DEZ 2014

SOUSA, C. M. M. G. de, GIL, E. P., SANTANA, L C. de. Caderno de Administração. Revista do Departamento de Administração da FEA ISSN 1414-7394 Pontifícia Universidade Católica de São Paulo Vol. 9, n.1. Jan - Dez. 2015, p. 73-84. 